\title{
Tenosynovial Giant Cell Tumor, Localized Type
}

National Cancer Institute

\section{Source}

National Cancer Institute. Tenosynovial Giant Cell Tumor, Localized Type. NCI

Thesaurus. Code C6532.

A well-circumscribed, lobulated tumor, completely or partially covered by a fibrous capsule. It usually arises in the fingers. It is characterized by the presence of mononuclear cells, multinucleated osteoclast-like giant cells, hemosiderin-laden macrophages, foam cells, and an inflammatory infiltrate. The tumor is slow-growing, usually developing over several years. Clinical presentation includes painless edema of the affected site. 